\title{
Rollen und Verantwortlichkeiten für erfolgreiche Social-Business-Anwendungen
}

\section{Handlungsempfehlungen für eine erfolgreiche Umsetzung in Unternehmen}

\author{
Christian Schiller und Thomas Meiren
}

\section{Zusammenfassung}

Der Einsatz von Social Business bietet große Potenziale für Unternehmen, ist jedoch kein Selbstläufer. Um auf Dauer von Social Business profitieren zu können, müssen deshalb von Beginn an erfolgskritische Fragestellungen adressiert und geeignete Lösungen gefunden werden. So ergab eine Studie, die im Rahmen des vom Bundesministerium für Bildung und Forschung (BMBF) geförderten Projekts SB:Digital durchgeführt wurde, dass die dort befragten Unternehmen klar definierte Rollen und Verantwortlichkeiten sowie die Sicherstellung der Akzeptanz der Mitarbeitenden als die wichtigsten Erfolgsfaktoren für Social Business ansehen. Innerhalb des Projekts SB:Digital wurde deshalb ein Referenzmodell entwickelt, das Unternehmen bei der Entwicklung und Umsetzung von Social-Business-Anwendungen unterstützt und dabei methodische und praktische Hilfestellungen bietet. Eine wichtige Funktion innerhalb des Referenzmodells nehmen klar definierte Rollen und Verantwortlichkeiten ein, welche im vorliegenden Beitrag vorgestellt werden.

\subsection{Einleitung}

Soziale Netzwerke fördern den Austausch und die Kollaboration verschiedener Akteure zum gegenseitigen Informations- und Wissensaustausch. Die dafür notwendigen Funktionen werden zunehmend über digitale Plattformen, wie bspw. soziale Medien, zur Verfügung gestellt. Dadurch haben sich in den letzten Jahren bereits tiefgreifende Auswirkun-

\section{Schiller $(\varangle) \cdot$ T. Meiren}

Fraunhofer IAO, Stuttgart, Deutschland

E-Mail: Christian.Schiller@iao.fraunhofer.de; thomas.meiren@iao.fraunhofer.de 
gen auf das gesellschaftliche Miteinander, insbesondere in der Kommunikation, ergeben. Auch Unternehmen haben inzwischen erkannt, dass sie sich diesem Trend nicht entziehen können und stellen sich dementsprechend zunehmend darauf ein. Neben der Kommunikation nach außen, bspw. zur Unterstützung von Marketing-Aktivitäten, spielt dabei auch die Unterstützung der Kommunikation im Inneren eines Unternehmens eine immer größere Rolle. Soziale Netzwerke können dabei helfen, effizientere und kollaborativere Verbindungen zwischen den Arbeitskräften zu ermöglichen, um Informationen und Daten auszutauschen (Kiron et al. 2012). Das hat Auswirkungen beispielsweise auf das Wissensmanagement in Unternehmen, welchem das Potenzial erwächst, kostengünstiger, allgegenwärtiger, standardisierter und mobiler zu werden, gleichzeitig personalisierter und effektiver im Hinblick auf die Erfüllung individueller Bedürfnisse (Krogh 2012). Auch das Innovationsmanagement eines Unternehmens kann davon profitieren, das soziale Netzwerk dient auch als ,Verstärker“ für Mitarbeiter*innen, die ansonsten kein Gehör finden würden (Recker et al. 2016). Die genannten Faktoren tragen allesamt dazu bei, Unternehmen auf dem Weg zu einem ,Social Business“ maßgeblich zu unterstützen. Social Business wird im Rahmen dieses Kapitels allgemein als Strategie und Rahmenwerk verstanden, mit dessen Anwendung die Generierung eines sozialen, ökologischen und ökonomischen Nutzens aus dem Einsatz digitaler sozialer Netzwerke als primärem Ziel verbunden wird.

Eine mittels digitaler sozialer Netzwerke optimierte interne Kommunikation kann einem Unternehmen u. a. folgende Vorteile bieten (Bruysten 2019):

- sie ist Treiber von Motivation und Innovation,

- sie kann als Frühwarnsystem für Verantwortliche im Unternehmen dienen,

- zuständige Experten und Abteilungen lassen sich schnell und einfach identifizieren,

- sie kann zu sinkenden Kosten und steigenden Umsatz und Gewinnen beitragen.

Viele Unternehmen gehen jedoch fälschlicherweise davon aus, dass sich interne Prozesse automatisch verändern und anpassen, sobald sie ihrer Belegschaft ein darauf ausgerichtetes digitales soziales Netzwerk zur Verfügung stellen. Allerdings muss ein Wandel der Kommunikationskultur immer auch Hand in Hand mit einem Wandel der Unternehmenskultur vonstattengehen (Fischer-Kienberger 2016). Um positive Auswirkungen auf den Output zu erlangen sind Leadership und strategisches Denken nötig (Hinchcliffe und Kim 2012). Es ist wichtig, sich Gedanken über Chancen und Risiken, über Erfolgsfaktoren und mögliche Gründe für ein Scheitern sozialer Netzwerke zu machen. Insbesondere müssen jedoch auch arbeitsorganisatorische Fragestellungen bedacht werden. Wer ist für die Einführung und Umsetzung eines solchen Netzwerkes verantwortlich? Wer organisiert die Kommunikation an die Mitarbeiter*innen? Und welche Aufgaben sind darüber hinaus notwendig, um ein digitales soziales Netzwerk erfolgreich am Laufen zu halten?

Diesen und weiteren Fragen möchte der folgende Beitrag nachgehen und Unternehmen möglichst praxisorientierte Handlungsempfehlungen mit auf den Weg geben. 


\subsection{Rollenkonzepte und Qualifikationsprofile}

Die laufende Digitalisierung und Automatisierung stellt viele Unternehmen vor große Herausforderungen, bietet aber auch große Chancen, egal ob beispielsweise durch Industrie 4.0, Smart Services oder Servicerobotik (Schuh et al. 2017; Freitag et al. 2019; Schiller et al. 2019a). Neben technischen sowie prozess- und informationstechnischen Aspekten stellen die Folgen der Digitalisierung Unternehmen jedoch auch in arbeitsorganisatorischen Fragestellungen vor große Herausforderungen. Mitarbeiter*innen aus unterschiedlichsten Bereichen, wie beispielsweise Entwicklung, Vertrieb und Service, müssen auf sich verändernde Prozesse mit Hilfe von passenden Qualifizierungsangeboten vorbereitet werden. Viele Unternehmen machen in dieser Hinsicht jedoch noch deutlich zu wenig, so sind beispielsweise neue Formen der Kompetenzvermittlung an vielen Stellen wünschenswert aber bisher noch nicht weit verbreitet (Schletz et al. 2017). Außerdem stellt sich für viele Unternehmen zunächst die Frage, wie sich Arbeit und die entsprechenden Qualifikationsanforderungen überhaupt konkret verändern (Hamann et al. 2019). Die Digitalisierung interner Prozesse spielt dabei eine wichtige Rolle (Borchert et al. 2019). Physische und virtuelle Räume verschwimmen immer mehr miteinander und neue Formen mobiler Arbeit entstehen (Leimeister et al. 2015). Digitale soziale Netzwerke sind ein mögliches Werkzeug, um interne Kommunikationsprozesse im virtuellen Raum zu ermöglichen und damit zum Gelingen mobiler Arbeitsformen beizutragen.

Im Rahmen des vom Bundesministerium für Bildung und Forschung (BMBF) geförderten und vom Projektträger Karlsruhe (PTKA) betreuten Projekts „Social Business Digitale soziale Netzwerke als Mittel zur Gestaltung attraktiver Arbeit (SB:Digital)“ wurde deshalb ein Referenzmodell entwickelt, das Unternehmen bei der Entwicklung und Umsetzung von Social-Business-Anwendungen unterstützt und dabei methodische und praktische Hilfestellungen bietet. Eine wichtige Funktion innerhalb des Referenzmodells nehmen klar definierte Rollen und Verantwortlichkeiten ein. Hierbei werden wichtige Rollen für Social-Business-Anwendungen identifiziert und die entsprechenden Aufgaben zugeordnet und detailliert beschrieben. Daraus lassen sich anschließend für jede Rolle die erforderlichen Kompetenzen ableiten und Qualifikationsprofile erstellen. Dabei ist zu beachten, dass der Begriff der „Rolle“ nicht mit einer „Stelle“ in Unternehmen gleichzusetzen ist. Vielmehr ist mit einer Rolle lediglich die Ausführung bestimmter Aufgaben verbunden - unabhängig von der hierarchischen Position der ausführenden Person im Unternehmen. Im folgenden Beitrag wird ein konkretes Rollenkonzept für Social Business in Unternehmen vorgestellt, das auf der Basis der Analyse von Stellenanzeigen, der Befragung von Expertinnen und Experten sowie einer im Rahmen des Projektes durchgeführten Breitenerhebung - die Befragung von über 132 Unternehmen unterschiedlichster Branchen und Betriebsgrößen entwickelt wurde. Um das Rollenkonzept insbesondere in kleinen und mittleren Unternehmen einsetzen zu können, wurde die Zahl der Rollen bewusst überschaubar gehalten. 


\subsubsection{Rollen}

Unter einer Rolle versteht man ,die Beschreibung einer Menge von Aufgaben und Verantwortlichkeiten im Rahmen eines Projektes und einer Organisation“ (V-Modell XT Bund 2010). Dabei sind „Rollen“ nicht mit „Stellen“ oder „Personen“ zu verwechseln. In der Regel kann eine Person mehrere Rollen besetzen. Außerdem sind Rollen häufig auch mehrfach, d. h. durch mehrere Personen oder gar Organisationseinheiten, besetzt.

Möchte man übersichtlich darstellen, welche Aufgaben in einem Projekt oder einem Tätigkeitsfeld durch welche Rollen übernommen werden, empfiehlt sich die Erstellung eines Rollenkonzepts, idealerweise in Form einer Matrix (siehe Abb. 9.1). Anwendung findet dieses Verfahren u. a. bereits in der Softwareentwicklung (V-Modell), im Service Engineering und im Zusammenhang mit der Entwicklung neuer Elektromobilitätskonzepte (Bröhl und Dröschel 1995; Meiren und Barth 2002; DIN SPEC 91364 2018).

Rollenkonzepte dienen der Spezifizierung und schaffen Transparenz über die Zuordnung von Aufgaben und Verantwortlichkeiten. Der Grad der Verantwortlichkeit der jeweiligen Rollen für die unterschiedlichen Aufgaben wird durch die Kennzeichnungen „A “ für ,,ausführend“, „M“ für „mitwirkend“ und „B“ für „,beratend“ transparent in der Matrix dargestellt. Dadurch unterstützen sie das Projektmanagement bei der Zusammensetzung von Projektteams sowie der Abschätzung von Personalbedarfen ebenso wie die Identifikation von Qualifizierungsbedarfen (Frings et al. 1999).

Ein weiterer Vorteil von Rollenkonzepten ist die Flexibilität bei der Zuordnung von Aufgaben zu konkreten Personen in der Organisation. Wie oben bereits beschrieben, liegt das insbesondere daran, dass eine Person meistens mehrere Rollen übernimmt, gleichzeitig jedoch auch viele Rollen von mehreren Personen ausgeübt werden (vgl. auch DIN SPEC 91364 2018). Eine Person ist also nicht fest an eine bestimmte Rolle gebunden. Stattdessen hat sie die Möglichkeit, flexible Rollen mit wechselnden Qualifikations- und Kompetenzanforderungen zu übernehmen. Damit wird es Unternehmen ermöglicht, zugleich wesentlich zielgerichteter mit vorhandenen und zukünftigen Humanpotenzialen umzugehen (Keith und Groten 2004).

Abb. 9.1 Beispiel eines Rollenkonzepts in Anlehnung an (Meiren und Barth 2002)

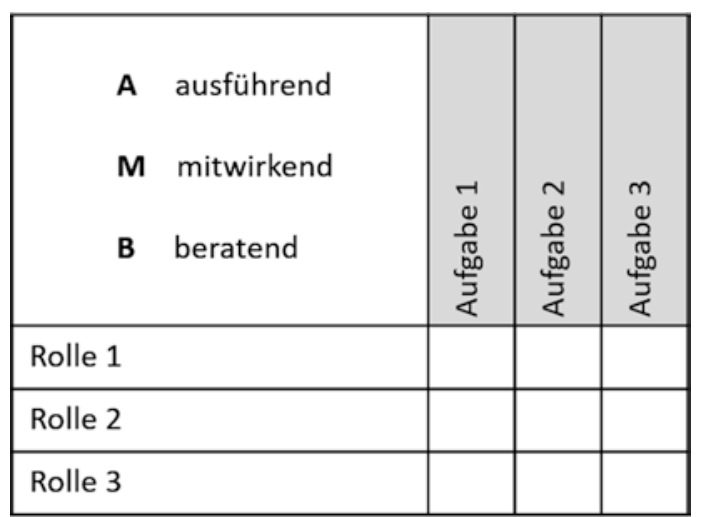


Um eine übersichtliche und möglichst umfangreiche Rollenbeschreibung zu verwirklichen, wird in der Literatur folgende Gliederung empfohlen (Meiren und Barth 2002; DIN SPEC 91364 2018; Keith und Groten 2004):

1. Namen:

Die Bezeichnungen der Rollen sind prinzipiell frei wählbar, sollten allerdings sinnvollerweise einen Bezug zu den mit der Rolle zusammenhängenden Aufgaben haben. Zudem sollten sie nicht zu abstrakt, sondern leicht verständlich sein.

2. Aufgaben und Ergebnisse:

Für jede Rolle ist klar zu definieren, wie die jeweiligen Aufgaben und Verantwortlichkeiten sind. Das ist ein wesentliches Abgrenzungsmerkmal der Rollen zueinander.

3. Kompetenzen und Qualifikationen:

Jede Rolle beinhaltet eine Beschreibung der für die Bearbeitung der rollenspezifischen Aufgaben notwendigen Kompetenzen und Qualifikationen. Näheres dazu siehe Abschn. 9.2.2.

4. Beziehungen der Rollen untereinander:

Rollen können Beziehungen zueinander haben, welche Informations- und Kommunikationsflüsse nach sich ziehen. Für die Bearbeitung eines Projektes ist es zielführend, diese möglichst transparent zu machen.

Da bei der Definition von Rollen die Erstellung von Qualifikations- und Kompetenzprofilen, neben der Festlegung von Verantwortlichkeiten für bestimmte Aufgaben, eine der Kernaufgaben darstellt, sollen diese im folgenden Abschnitt tiefgehender erläutert werden.

\subsubsection{Qualifikationsprofile}

Ein Profil bildet laut Duden eine „Gesamtheit von Eigenschaften ab, die unverwechselbar typisch für jemanden oder etwas sind“. Dementsprechend werden in einem Qualifikationsprofil für eine vorher definierte Rolle diejenigen Qualifikationseigenschaften dargestellt, die jeweils charakteristisch für diese sind und zur erfolgreichen Bearbeitung der zugeteilten Aufgaben hilfreich oder gar erforderlich sein können. Allerdings können die Qualifikationsprofile der unterschiedlichen Rollen inhaltlich nicht immer disjunkt voneinander betrachtet werden, anders als die (ausführende) Verantwortlichkeit der Rollen. Die Abgrenzung der Rollen zueinander erfolgt deshalb eher in der Summe der Qualifikationseigenschaften und im jeweiligen Grad der Verantwortlichkeit. Formal bedeutet dies für die Beziehung von jeweiliger Rolle (R) zum entsprechenden Qualifikationsprofil (Q) folgendes:

$$
Q \subseteq R: \Leftrightarrow \forall x \in Q: x \in R
$$


Dagegen ist die ausführende Verantwortung (in Abschn. 9.2.1 mit „A“ bezeichnet) einer Rolle $1\left(\mathrm{~V}_{\mathrm{R} 1}\right)$ und einer Rolle $2\left(\mathrm{~V}_{\mathrm{R} 2}\right)$ bezüglich einer Aufgabe klar voneinander abgegrenzt. Sie ist ein wesentliches Unterscheidungsmerkmal zwischen den Rollen und lässt sich wie folgt formal darstellen:

$$
V_{R 1} \cap V_{R 2}=\varnothing
$$

Die Qualifikationsprofile $\mathrm{Q}_{1}$ und $\mathrm{Q}_{2}$ der jeweiligen Rollen können jedoch durchaus gemeinsame Schnittmengen haben:

$$
\cap\left\{Q_{1}, Q_{2}\right\}=\left\{x \mid\left(x \in Q_{1}\right) \wedge\left(x \in Q_{2}\right)\right\}
$$

Es bietet sich an, die Qualifikationen der Rollen in unterschiedliche Kategorien zu gliedern. Dies sorgt für eine bessere Übersichtlichkeit und Strukturierung des Qualifikationsprofils. In praxisorientierten Veröffentlichungen ist eine Unterteilung in fachliche, methodische und soziale Qualifikationen üblich (Meiren und Barth 2002; DIN SPEC 91364 2018). Als Darstellungsform wird eine Tabelle empfohlen (siehe Abb. 9.2).

Unter Fachkompetenzen versteht man diejenigen Fertigkeiten und Kenntnisse, welche der Inhaber einer Rolle zur erfolgreichen und professionellen Bearbeitung der rollenspezifischen Aufgaben mitbringen sollte. Methodenkompetenzen geben Auskunft über die Fähigkeit zur systematischen Problemlösung in unterschiedlichen Kontexten. Unter Sozialkompetenzen werden die notwendigen Fähigkeiten einer Rolle zur effektiven Bewältigung der aufgabenspezifischen Kommunikations- und Interaktionsprozesse zusammengefasst.

\subsection{Erstellung eines Rollenkonzeptes für Social Business}

Zur Erstellung des Rollenkonzeptes und verschiedener Qualifikations- und Kompetenzprofile wurden unterschiedliche Quellen herangezogen und als Ausgangsbasis verwendet. Wichtigste Quelle ist die im Rahmen des Projekts SB:Digital durchgeführte Breitenerhebung unter 132 Unternehmen aus Deutschland. Neben den Chancen und Risiken, welche sich für Unternehmen durch den Einsatz interner sozialer Netzwerke ergeben, wurden hier auch die wichtigsten Erfolgsfaktoren und die häufigsten Gründe für ein Scheitern abge-

\begin{tabular}{|l|l|l|}
\hline Fachlich & Methodisch & Sozial \\
\hline Fachkompetenz 1 & Methodenkompetenz 1 & Sozialkompetenz 1 \\
\hline Fachkompetenz 2 & Methodenkompetenz 2 & Sozialkompetenz 2 \\
\hline$\ldots$ & $\ldots$ & $\ldots$ \\
\hline Fachkompetenz $\mathrm{n}$ & Methodenkompetenz $\mathrm{n}$ & Sozialkompetenz $\mathrm{n}$ \\
\hline
\end{tabular}

Abb. 9.2 Beispiel für ein Qualifikationsprofil (eigene Darstellung) 
fragt. Eine weitere wichtige Quelle zur Erstellung des Rollenmodells waren die Ergebnisse mehrerer von einem Projektpartner durchgeführten Experteninterviews. Dazu wurden fünf Experten mittels teilstrukturierter Fragebögen offen befragt. Die Ergebnisse von Breitenerhebung und Experteninterviews liefern zusammen die wesentliche Ausgangsbasis zur Formulierung von Aufgaben in einem Social Business.

Zur weiteren Detaillierung des Rollenkonzepts und der Qualifikationsprofile wurden über die genannten Erhebungen hinaus über 300 Stellenanzeigen aus dem Bereich ,Social Media“ analysiert. Die Stellenanzeigen wurden auf verschiedenen Jobbörsen, wie beispielsweise Stepstone, indeed und Xing recherchiert.

\subsubsection{Definition der Aufgaben}

Wie oben bereits erwähnt, bildete die im Rahmen von „SB:Digital“ durchgeführte Breitenerhebung und die Experteninterviews die Ausgangsbasis zur Formulierung von Aufgaben für die zu definierenden Rollen für eine erfolgreiche Umsetzung eines internen und digitalen sozialen Netzwerkes. Im Folgenden sollen daher die in diesem Zusammenhang zentralen Aspekte dieser Erhebungen zusammengefasst und die sich daraus ergebenden Aufgaben hergeleitet werden.

Als besonders wichtig wird die Wahrnehmung potenzieller Chancen eingeschätzt (Schiller et al. 2019b; Schiller und Meiren 2018). Laut den Ergebnissen der durchgeführten Breitenerhebung unter 132 Unternehmen in Deutschland werden diese insbesondere in einer Verbesserung der Zusammenarbeit (Anforderungsnummer 1), in einer spürbaren Eindämmung an E-Mails (2) und in einer engeren Einbindung der Mitarbeiter*innen in wichtige Unternehmensentscheidungen (3) gesehen.

Weitere wichtige Punkte sind ein strukturiertes Vorgehen und die Definition klarer Rollen und Verantwortlichkeiten bei der Einführung und Umsetzung eines sozialen Netzwerkes im Unternehmen (4). Dadurch können bestehenden Ängsten, beispielsweise vor einer zu starken Vermischung von Privatleben und Arbeit, vor Datenmissbrauch und vor einem Gefühl der Überwachung, entgegengewirkt werden. Strukturiertes Vorgehen unterstützt auch die erforderliche Schaffung von Transparenz.

Die Akzeptanz unter den Angestellten zu fördern ist eines der wichtigsten Aspekte und Erfolgsfaktoren bei der Einführung interner sozialer Netzwerke. Dazu erforderlich ist eine professionelle und an den Bedürfnissen der Beschäftigten ausgerichtete Umsetzung (5), für die Mitarbeitenden interessante Inhalte (6) und die Schaffung von Feedback-Kanälen für die Mitarbeitenden (7). Im Einzelfall kann auch die Einführung von Incentives in einem Unternehmen sinnvoll sein.

Die zentralen Aspekte aus den Experteninterviews werden im Folgenden dargestellt (ebenfalls nach Schiller, Zinke-Wehlmann et al. 2019b); wichtig ist es demnach, eine geeignete Unternehmenskultur (8) zu fördern, in der sich Führungskräfte und Angestellte auf Augenhöhe begegnen. Die Arbeitsorganisation sollte ergebnis- und nicht präsenzorientiert sein. Einer der entscheidenden Erfolgsfaktoren für den Einsatz unternehmensinterner 
sozialer Netzwerke und das damit einhergehende selbstbestimmte Arbeiten ist die individuelle Motivation der Belegschaft. Es sollte bei den Mitarbeitenden ein gewisses Grundverständnis darüber geschaffen werden, wie interne soziale Netzwerke funktionieren, welche Potenziale diese haben und wie wichtig ihr eigenes Engagement zur erfolgreichen Umsetzung ist. Jeder sollte befähigt werden, soziale Netzwerke für sich selber nutzenbringend einzusetzen. Dementsprechend sollten Unternehmen Schulungs- und Weiterbildungsformate entwickeln, um ihren Arbeitskräften die entsprechenden Kompetenzen zu vermitteln (9). Wichtig sind auch flache Hierarchien und wenig Bürokratie. Führung sollte als Strukturgeber fungieren und dazu beitragen, dass alle an den Unternehmenszielen partizipieren und diese eigenständig voranbringen. Die Eigenständigkeit der Mitarbeiter*innen sollte dementsprechend gefördert werden (10).

Für die Experten ist es besonders wichtig, dass eine passende Infrastruktur für die Nutzung von sozialen Netzwerken geschaffen wird, was insbesondere eine weitgehende Systemintegration und die Möglichkeit einer direkten Kommunikation bedeutet (11).

Eine wichtige Aufgabe der Unternehmen ist es, den Nutzen von internen sozialen Netzwerken darzustellen und ein Problembewusstsein zu erzeugen. Das kann am ehesten durch Transparenz und entsprechende Handlungsleitfäden erzeugt werden (12). Auch Erfolgsgeschichten anderer Angestellten sollten regelmäßig kommuniziert werden. Eine entsprechende Kommunikationskampagne sollte von daher Bestandteil einer erfolgreichen Umsetzungsstrategie sein (13).

Die wichtigsten Aufgaben bei der Einführung eines internen sozialen Netzwerkes sind in Abb. 9.3 übersichtlich dargestellt.

Die Aufgaben 14 bis 18 ergänzen die aus den Anforderungen aus der Breitenerhebung und den Experteninterviews abgeleiteten Aufgaben. Sie stammen aus der Analyse von Stellenanzeigen aus bekannten Jobbörsen im Internet (siehe oben).

\subsubsection{Definition der Rollen}

Nach Herleitung der wichtigsten Aufgaben wurden im Rahmen zweier Workshops verschiedene Rollen festgelegt, um die Verantwortlichkeit für die jeweiligen Aufgaben klar zu definieren. Diese Rollen sind:

- Social Business Manager

- Content Manager

- Entwickler

- Kommunikationsmanager

- Mitarbeiter*innen

- Führungskraft

Die Zuordnung der Rollen zu den in Abschn. 9.3.1 hergeleiteten Aufgaben erfolgt über eine Matrix. Diese ist das zentrale Element im Rollenkonzept (siehe Abb. 9.4). Wie oben bereits erläutert, bedeutet „A“ „,ausführend“, „B“ „,beratend“ und „,M“ „,mitwirkend“. Die 


\begin{tabular}{|c|c|c|c|c|}
\hline $\begin{array}{l}\text { Anf. } \\
\text { Nr. }\end{array}$ & Aufgaben & $\begin{array}{l}\text { Breiten- } \\
\text { erhebung }\end{array}$ & $\begin{array}{l}\text { Experten- } \\
\text { interviews }\end{array}$ & $\begin{array}{l}\text { Stellen- } \\
\text { anzeigen }\end{array}$ \\
\hline 1 & Kollaborationskanäle schaffen & $\mathrm{x}$ & & \\
\hline 2 & Chatmöglichkeiten schaffen und verwalten & $\mathrm{x}$ & & \\
\hline 3 & Mitarbeiterpartizipation ermöglichen & $\mathrm{x}$ & & \\
\hline 4 & $\begin{array}{l}\text { Social-Business-Konzept und -Strategie } \\
\text { erstellen }\end{array}$ & $x$ & & \\
\hline 5 & Mitarbeiterbedürfnisse erfassen & $\mathrm{x}$ & & \\
\hline 6 & interessante Inhalte kontinuierlich erstellen & $\mathrm{x}$ & & \\
\hline 7 & Feedback-Kanäle schaffen und verwalten & $\mathrm{x}$ & & \\
\hline 8 & passende Unternehmenskultur fördern & & $x$ & \\
\hline 9 & Schulungsformate entwickeln und durchführen & & $\mathrm{x}$ & \\
\hline 10 & Eigenständigkeit der Mitarbeiter fördern & & $\mathrm{x}$ & \\
\hline 11 & $\begin{array}{l}\text { passende Infrastruktur schaffen } \\
\text { (Systemintegration, direkte Kommunikation) }\end{array}$ & & $\mathrm{x}$ & \\
\hline 12 & $\begin{array}{l}\text { Handlungsleitfäden und Best-Practice-Berichte } \\
\text { für interne Kommunikation erstellen }\end{array}$ & & $\mathrm{x}$ & \\
\hline 13 & $\begin{array}{l}\text { Kommunikationskampagnen planen und } \\
\text { durchführen }\end{array}$ & & $x$ & \\
\hline 14 & Analysen und Reportings erstellen & & & $x$ \\
\hline 15 & neue digitale Formate entwickeln & & & $x$ \\
\hline 16 & soziales Netzwerk betreuen und optimieren & & & $x$ \\
\hline 17 & Informationsrecherche für relevante Inhalte & & & $x$ \\
\hline 18 & Fragen der Community beantworten & & & $\mathrm{x}$ \\
\hline
\end{tabular}

Abb. 9.3 Wichtige Aufgaben in Unternehmen bei der Einführung eines internen sozialen Netzwerkes

Hauptverantwortung über die jeweilige Aufgabe hat immer die ,ausführende“ Rolle. Die zweithöchste Verantwortungsstufe ist „mitwirkend“. Diese Rollen sind direkt um eine Mitwirkung bei der Bewerkstelligung der Aufgabe angehalten und die ausführende Rolle zu unterstützen. Bei Bedarf unterstützt darüber hinaus noch die beratende Rolle. Dies geschieht entweder auf Anfrage durch die ausführende Rolle oder falls die beratende Rolle eine relevante Information zur Bewältigung der Aufgabe erhält.

\subsubsection{Herleitung der Qualifikationen und Kompetenzen}

Wie in Abschn. 9.2.1 dargestellt, beinhaltet eine vollständige Rollenbeschreibung auch Angaben über empfohlene Kompetenzen und Qualifikationen. Zur Strukturierung sind diese in fachliche, methodische und soziale Kompetenzen unterteilt. Die für jede Rolle erforderlichen Qualifikationen und Kompetenzen ergeben sich aus den zu bewältigenden 


\begin{tabular}{|c|c|c|c|c|c|c|c|c|c|c|c|c|c|c|c|c|c|c|}
\hline $\begin{array}{l}\text { A ausführend } \\
\text { M mitwirkend } \\
\text { B beratend }\end{array}$ & 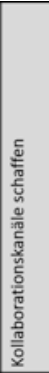 & 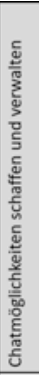 & 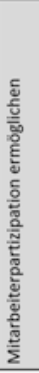 & 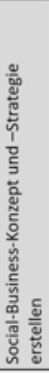 & 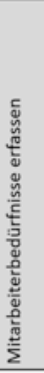 & 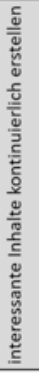 & 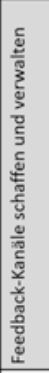 & 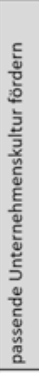 & 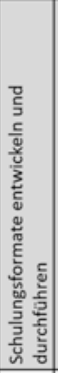 & 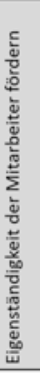 & 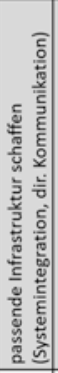 & 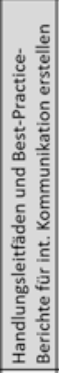 & 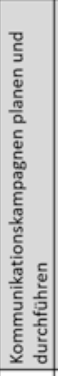 & 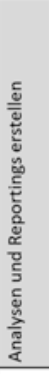 & 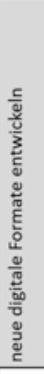 & 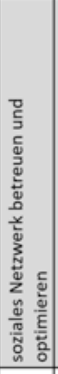 & 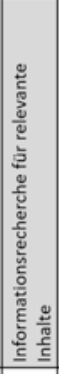 & 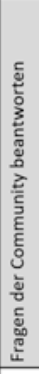 \\
\hline Social Business Manager & A & B & B & A & B & M & M & B & A & & M & B & B & A & B & A & M & B \\
\hline Content Manager & & & & & & A & & & & & & & & & A & B & A & B \\
\hline Entwickler & M & A & M & & & & M & & B & & A & & & B & & & & \\
\hline Kommunikationsmanager & в & & & B & A & M & A & & & & & A & A & & & & B & A \\
\hline Mitarbeiter & & & & & M & & & M & M & M & & & & & & & & \\
\hline Führungskraft & & & A & $M$ & M & B & & A & M & A & & & & & & & & \\
\hline
\end{tabular}

Abb. 9.4 Rollenkonzept für die erfolgreiche Anwendung interner sozialer Netzwerke in Unternehmen

Aufgaben und geben lediglich ein Idealbild ab. Bei der Zuordnung von konkreten Personen zu den Rollen kann es dabei in der Realität zu Abweichungen kommen. Sind diese Abweichungen zu groß, muss von der verantwortlichen Führungskraft entschieden werden, ob eine Weiterbildung der ausgewählten Person oder eine Neuzuordnung der Rolle zu einer anderen Person die bessere Alternative ist.

Im Folgenden soll am Beispiel der Rolle des Social Business Managers ein entsprechendes Qualifikationsprofil hergeleitet werden.

Fachlich: In Stellenanzeigen mit vergleichbaren Aufgaben, wie beispielsweise „Social Media Manager“, wird in den allermeisten Fällen ein abgeschlossenes Studium vorausgesetzt. Im Idealfall sollte dieses Studium im Bereich Medien, Marketing oder Kommunikation erfolgt sein. Das passt auch sehr gut mit den in Abschn. 9.3.1 definierten Aufgaben und den in Abschn. 9.3.2 zugeordneten (Haupt)Verantwortungsbereichen für die Rolle „Social Business Manager“" zusammen.

In den letzten Jahren gab es zunehmend Diskussionen um den Datenschutz. Spätestens nach Einführung der DSGVO im Mai 2018 ist das Thema in den meisten Unternehmen auf die Tagesordnung gekommen. Bei der Bewertung der Risiken durch den Einsatz interner sozialer Netzwerke in Unternehmen im Rahmen der Breitenerhebung in SB:Digital wurde das Risiko eines „Datenmissbrauchs“ am zweithöchsten bewertet. Dementsprechend ist es für die Rolle eines „Social Business Managers“ empfehlenswert, wenn die ausführende Person bereits Erfahrungen mit dem Thema Datenschutz aufweisen kann. Dadurch kann auch in der Kommunikation an die Mitarbeiter*innen eine Sensibilisierung bezüglich dieses Themas signalisiert werden.

Die weiteren fachlichen Qualifikationen runden das Profil ab. So sind Branchenkenntnisse, Kenntnisse in der Informationstechnik und Coaching Erfahrungen wünschenswert. 
Methodisch: Da die Rolle eines „Social Business Managers“ eine Vielzahl an Aufgaben hat und zudem schwerpunktmäßig mit Strategie- und Analyseaufgaben beschäftigt ist, sind ein strukturiertes Vorgehen und ein lösungsorientiertes Herangehen an Probleme erforderlich. Auch methodische Kenntnisse in Analyse und Statistik sind zur Bewältigung dieser Aufgaben äußerst wichtig. Darüber hinaus sollte natürlich die Fähigkeit vorhanden sein, verschiedene Social Media Tools bedienen und vergleichen zu können.

Empfehlenswert sind zudem Kenntnisse in Projekt-, Prozess- und Qualitätsmanagement, da man in der Rolle eines ,Social Business Managers“ das soziale Netzwerk betreut und optimiert und damit auch die Verantwortung über die Prozesse und Qualität sowie die erfolgreiche Umsetzung von Projekten in diesem Umfeld trägt.

Wünschenswert ist es, wenn Kenntnisse über agile Methoden, Wissensmanagement, Changemanagement sowie Netzwerkmanagement vorhanden sind.

Sozial: Da für die Rolle eines „Social Business Managers“ durchaus eine Führungskraft oder eine Stabsstelle denkbar ist und er zudem als Spezialist und zentraler Ansprechpartner für dieses Thema im Unternehmen gilt, ist ein hohes Maß an Selbstmanagement sowie Durchsetzungsfähigkeit dringend notwendig. Wichtig sind zudem ein hohes Maß an Kommunikationsfähigkeit, Teamfähigkeit und Kundenorientierung. Zu beachten ist, dass „Kunden“ in diesem Fall die eigenen Mitarbeiter*innen im Unternehmen sind.

Abgerundet werden die sozialen Qualifikationen durch ein ausreichendes Maß an Berufserfahrung und durch Fremdsprachenerfahrung. Ist der „Social Business Manager“ auch Standorte in nicht-deutschsprachigen Ländern zuständig, sind Fremdsprachenerfahrung deutlich höher priorisiert und ebenfalls zwingend notwendig.

In Abb. 9.5 sind die für die Rolle „Social Business Manager“ definierten Qualifikationen und Kompetenzen zusammengefasst dargestellt.

\begin{tabular}{|l|l|l|}
\hline Fachlich & Methodisch & Sozial \\
\hline $\begin{array}{l}\text { Abgeschlossenes Studium } \\
\text { (Medien, Marketing, } \\
\text { Kommunikation) }\end{array}$ & $\begin{array}{l}\text { Strukturiertes Vorgehen und Herangehen } \\
\text { an Probleme }\end{array}$ & Selbstmanagement \\
\hline Erfahrungen mit Datenschutz & Bedienung von Social Media Tools & Durchsetzungsfähigkeit \\
\hline Branchenkenntnisse & Analyse und Statistik & Kommunikationsfähigkeit \\
\hline $\begin{array}{l}\text { Kenntnisse in } \\
\text { Informationstechnik }\end{array}$ & Projektmanagement & Teamfähigkeit \\
\hline Coaching Erfahrungen & Prozessmanagement & Kundenorientierung \\
\hline & Qualitätsmanagement & Berufserfahrung \\
\hline & Kenntnisse zu agilen Methoden & Fremdsprachenerfahrung \\
\hline & Wissensmanagement & \\
\hline & Changemanagement & \\
\hline
\end{tabular}

Abb.9.5 Qualifikations- und Kompetenzprofil für einen Social Business Manager 
Das hergeleitete Qualifikations- und Kompetenzprofil ist bewusst relativ allgemein formuliert worden. Je nach Branche oder Unternehmen ändern sich die konkreten Aufgaben und damit auch die erforderlichen Qualifikationen und Kompetenzen oder müssen ergänzt werden. Abhängig ist die konkrete Ausformulierung des Rollenkonzepts und des Qualifikations- und Kompetenzprofils zudem von der jeweiligen Organisationsform des betroffenen Unternehmens.

\subsubsection{Beziehungen zwischen den Rollen}

Die Darstellung der Beziehungen zwischen den einzelnen Rollen ist ein wesentlicher Bestandteil einer Rollenbeschreibung (Keith und Groten 2004). Dadurch werden mögliche Schnittstellen besser identifiziert und die Aufgaben- und Verantwortungsbereiche der einzelnen Rollen weiter geschärft.

Im hergeleiteten Rollenkonzept nimmt der „Social Business Manager“ die zentrale und koordinierende Rolle ein. Die Rollen des „Entwicklers“ und des „Content Managers“ bilden gewissermaßen das Back-Office und setzen die Konzepte des ,Social Business Managers" technisch und inhaltlich um. Dabei sollten sie ein größtmögliches Maß an Freiheiten genießen, um auch eigenständig und initiativ Verbesserungsvorschläge an den ,Social Business Manager"weiterzuleiten.

Die Schnittstelle zwischen Front-Office und „Social Business Manager“ bildet die Rolle des „Kommunikationsmanagers“. Er kommuniziert sowohl relevante Informationen und Neuigkeiten vom „Social Business Manager“ und aus dem Back-Office nach außen. Auf der anderen Seite fungiert er als Sprachrohr der Angestellten aus der Front-Office nach Innen. Zu beachten ist allerdings, dass sich sowohl Angestellte als auch Führungskräfte auch direkt an den ,Social Business Manager“ wenden können sollten. Damit dieser sich jedoch auf seine Hauptaufgaben konzentrieren kann, sollte die Hauptschnittstelle jedoch der „Kommunikationsmanager“ sein.

Die Rollen im Front-Office umfassen die Aufgaben, welche Angestellte und Führungskräfte im Rahmen ihrer Tätigkeiten im internen sozialen Netzwerk wahrnehmen. In der Rolle des „Mitarbeiters“ geht es insbesondere darum, das soziale Netzwerk auch zu nutzen und gegebenenfalls konstruktives Feedback zu geben. Die Rolle der „Führungskraft“ hat darüber hinaus auch noch die Aufgabe des Promotors und sie muss für Einführung und Förderung einer geeigneten Unternehmenskultur sorgen (Abb. 9.6).

\subsection{Abschließende Betrachtung}

An dieser Stelle möchten wir zum Abschluss unseres Beitrages noch einmal kurz zusammenfassen, wie wir dank der Ergebnisse der im Rahmen des Projekts „SB:Digital“ erhobenen Studie zum internen Einsatz von sozialen Netzwerken in Unternehmen die Fragen nach den Chancen und Risiken, sowie möglichen Gründen für ein Scheitern der Implementie- 


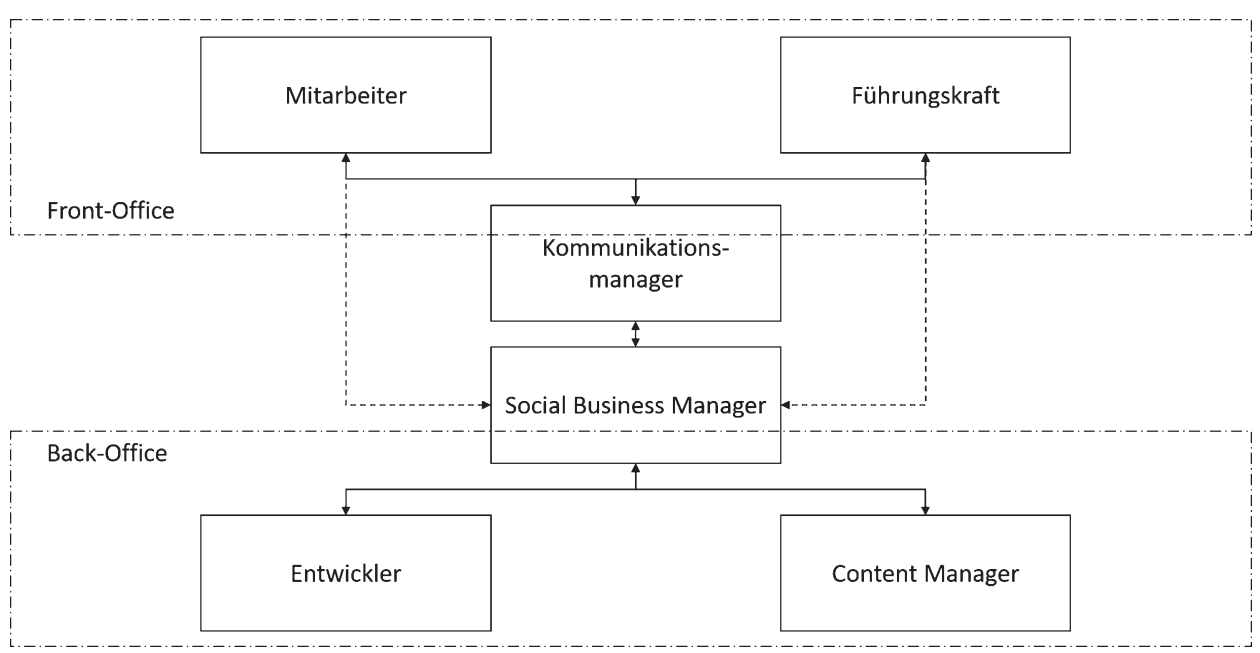

Abb. 9.6 Beziehungen zwischen den einzelnen Rollen

rung von sozialen Netzwerken auf konkrete Faktoren festlegen konnten. Anhand der Auswertung waren neben der Akzeptanz seitens der Mitarbeiter*innen die Festlegung klarer Rollen und Verantwortlichkeiten, der am häufigsten genannte Erfolgsfaktor (Schiller et al. 2019b). Zur Erarbeitung praxisorientierter Handlungsempfehlungen konzipierten wir ein Rollenkonzept, welches eine Hilfestellung bei der Umsetzung eines Social-Business-Ansatzes durch den Einsatz von internen sozialen Netzwerken geben soll. Des Weiteren lassen sich mit dem erarbeiteten Rollenkonzeptes auch die anfänglich definierten arbeitsorganisatorischen Fragestellungen klären. Wie beispielsweise, wer ist für die Umsetzung von Social-Business-Anwendungen zuständig, wer organisiert die interne Kommunikation und welche Qualifikationen sind überhaupt notwendig, um ein Enterprise Social Network erfolgreich am Laufen zu halten? Mit der Beantwortung dieser Fragen war es möglich ein entsprechendes Anforderungsprofil für das generierte Rollenkonzept zu definieren. Die Herleitung der wesentlichen Aufgaben, die zur erfolgreichen Umsetzung und Betrieb zu berücksichtigen sind, konzentrierte sich auf die Auswertungen der Studie (Experteninterviews und Breitenerhebung), sowie der Analyse von 306 Stellenanzeigen.

Nach der Definition von Rollen wurde mit Hilfe einer Zuordnungsmatrix ein Rollenkonzept aufgestellt, um die verschiedenen Tätigkeiten und Aufgaben den einzelnen Rollen zuzuordnen. Maßgeblicher Bestandteil eines solchen Konzeptes ist die Festlegung der jeweiligen Verantwortlichkeit, also ob jemand ,,ausführende“, „mitwirkende“ oder lediglich „,beratende“ Verantwortung für die jeweilige Aufgabe hat. Für jede Aufgabe sollte es nur eine Rolle ,ausführender“ Verantwortung geben. Das sorgt für Klarheit und Transparenz.

Auf Basis des Rollenkonzeptes wurden Qualifikations- und Kompetenzprofile hergeleitet, welche die Personalplanung bei der Zuordnung von konkreten Personen auf die verschiedenen Rollen unterstützen. 
Sowohl bei dem in diesem Kapitel vorgestellten Rollenkonzept als auch bei dem vorgestellten Qualifikations- und Kompetenzprofil ist die allgemeine Formulierung zu berücksichtigen. Je nach Unternehmen und Branche sind unter Umständen Ergänzungen und Anpassungen notwendig. Zudem ist die strikte Trennung von „Rollen“ und „Stellen“, beziehungsweise „Personen“, zu beachten. So kann beispielsweise die Rolle des „Social Business Managers“ entweder von einer tatsächlich ausschließlich dafür ausgewählten Person übernommen werden oder von jemandem, der zusätzlich noch eine oder mehrere andere Rollen einnimmt, wie die Rolle des Leiters (internes) Marketing. Dementsprechend variiert allerdings auch die Kapazität, die individuell dafür aufgewendet werden kann. So ist es in einem zusätzlichen Schritt durchaus empfehlenswert, die in Abschn. 9.3.1 hergeleiteten Aufgaben mit unternehmensspezifischen Prozessen zu hinterlegen und auf dieser Basis die notwendigen Kapazitäten je Rolle zu kalkulieren.

\section{Literatur}

Borchert, M., Martinez, S., Bienzeisler, Mohr, O., Fregin, M. C., Becker, S., Schmidt, K., Straub, M., \& Troch, S. L. (2019). Digitalisierung der Arbeitswelt in kommunalen Unternehmen. In W. Bauer, S. Stowasser, S. Mütze-Niewöhner, C. Zanker \& K. H. Brandl (Hrsg.), Arbeit in der digitalisierten Welt: Stand der Forschung und Anwendung im BMBF-Förderschwerpunkt (S. 202-209). Stuttgart: Fraunhofer IAO.

Bröhl, A. P., \& Dröschel, W. (1995). Das V-Modell: der Standard für die Softwareentwicklung mit Praxisleitfaden (2. Aufl.). München: Oldenbourg Verlag.

Bruysten, T. (2019). Interne Kommunikation: Social Media Learnings für Unternehmensnetzwerke. Online. http://bruysten.com/unternehmensberatung/interne-kommunikation-social-media-learnings-fur-unternehmensnetzwerke/. Zugegriffen am 31.01.2019.

DIN SPEC 91364. (2018). Leitfaden für die Entwicklung neuer Dienstleistungen zur Elektromobilität.

Fischer-Kienberger, C. (2016). Kommunikationskultur als Voraussetzung für erfolgreiche Geschäftsmodellinnovationen. In H. Granig et al. (Hrsg.), Geschäftsmodellinnovationen (S. 133-144). Wiesbaden: Springer/Gabler. https://doi.org/10.1007/978-3-658-08623-7_9.

Freitag, M., Korb, T., \& Sommer, P. (2019). Smart Services im Maschinen- und Anlagenbau - Eine Kurzstudie. Stuttgart: Fraunhofer IRB.

Frings, S., Weisbecker, A., Lahr, W., \& Reinsch, V. (1999). Rollenkonzept in der SoftwareEntwicklung. In U. Arend, E. Eberleh \& K. Pitschke (Hrsg.), Software-Ergonomie '99: Design von Informationswelten. Berichte des German Chapter of the ACM (Bd. 53, S. 73-84). Stuttgart/ Leipzig: Teubner.

Hamann, K., Link, M., Dworschak, B., \& Schnalzer, K. (2019). Auswirkungen der Digitalisierung auf Arbeit und Kompetenzentwicklung. In W. Bauer, S. Stowasser, S. Mütze-Niewöhner, C. Zanker \& K. H. Brandl (Hrsg.), Arbeit in der digitalisierten Welt. Stand der Forschung und Anwendung im BMBF-Förderschwerpunkt (S. 10-14). Stuttgart: Fraunhofer IAO.

Hinchcliffe, D., \& Kim, P. (2012). Social business by design: Transformation social media strategies for the connected company. San Francisco: Jossey-Bass.

Keith, H., \& Groten, C. (2004). Rollenkonzepte als moderne Instrumente für das Personalmanagement in Dienstleistungsprojekten. In H. Luczak, R. Reichwald \& D. Spath (Hrsg.), Service 
Engineering in Wissenschaft und Praxis - Die ganzheitliche Entwicklung von Dienstleistungen (S. 61-93). Wiesbaden: Deutscher Universitäts-Verlag/GWV Fachverlage GmbH.

Kiron, D., Palmer, D., Phillips, A. N., \& Kruschwitz, N. (2012). Social business: What are companies really doing? MIT Sloan Management Review. North Hollywood: Massachusetts Institute of Technology.

Krogh, G. (2012). How does social software change knowledge management? Toward a strategic research agenda. Journal of Strategic Information Systems, 21(2), 154-164. https://doi. org/10.1016/j.jsis.2012.04.003.

Leimeister, J. M., Zogaj, S., Durward, D., \& Blohm, I. (2015). Arbeit und IT: Crowdsourcing und Crowdwork als neue Arbeits- und Beschäftigungsformen. In verdi - Vereinte Dienstleistungsgewerkschaft. Gute Arbeit und Digitalisierung: Prozessanalysen und Gestaltungsperspektiven für eine humane digitale Arbeitswelt (S. 66-79). ver.di.

Meiren, T., \& Barth, T. (2002). Service Engineering in Unternehmen umsetzen - Leitfaden für die Entwicklung von Dienstleistungen. Stuttgart: Fraunhofer IRB.

Recker, J., Malsbender, A., \& Kohlborn, T. (2016). Using enterprise social networks as innovation platforms. IT Professional, 18(2), 42-49. https://doi.org/10.1109/MITP.2016.23.

Schiller, C., \& Meiren, T. (2018). Enterprise social networks for internal communication and collaboration - Results of an empirical study. Conference proceedings, 2018 IEEE International Conference on Engineering, Technology and Innovation (ICE/ITMC), Stuttgart. S. 787-790.

Schiller, C., Graf, B., Fischbach, J., Baumgarten, S., Bläsing, D., Strunck, S., Fredl-Maurer, R. \& Filitz, G. (2019a). Servicerobotik bei personenbezogenen Dienstleistungen - Abschlussbroschüre. Stuttgart: IAT Universität Stuttgart.

Schiller, C., Zinke-Wehlmann, C., Meiren, T., Friedrich, J., \& Holze, J. (2019b). Social Business Studie über den Einsatz interner sozialer Netzwerke in Unternehmen. Stuttgart: Fraunhofer.

Schletz, A., Martinetz, S., Wilke, J., Brzoska, S., Robers, D. I., Kaiser, S., Bähner, J., Baierl, M., Ludwig, F., \& Frey, C. (2017). Flexibilisierung von Personal- und Kompetenzmanagement im digitalen Wandel. Stuttgart: Fraunhofer-Institut für Arbeitswirtschaft und Organisation.

Schuh, G., Anderl, R., Gausemeier, J., ten Hompel, M., \& Wahlster, W. (2017). Industrie 4.0 Maturity Index. Die digitale Transformation von Unternehmen gestalten (acatech Studie). München: Herbert Utz.

V-Modell XT Bund. (2010). Teil 1: Grundlagen des V-Modells, Version 1.0 (Basis V-Modell XT 1.3).

Open Access Dieses Kapitel wird unter der Creative Commons Namensnennung 4.0 International Lizenz (http://creativecommons.org/licenses/by/4.0/deed.de) veröffentlicht, welche die Nutzung, Vervielfältigung, Bearbeitung, Verbreitung und Wiedergabe in jeglichem Medium und Format erlaubt, sofern Sie den/die ursprünglichen Autor(en) und die Quelle ordnungsgemäß nennen, einen Link zur Creative Commons Lizenz beifügen und angeben, ob Änderungen vorgenommen wurden.

Die in diesem Kapitel enthaltenen Bilder und sonstiges Drittmaterial unterliegen ebenfalls der genannten Creative Commons Lizenz, sofern sich aus der Abbildungslegende nichts anderes ergibt. Sofern das betreffende Material nicht unter der genannten Creative Commons Lizenz steht und die betreffende Handlung nicht nach gesetzlichen Vorschriften erlaubt ist, ist für die oben aufgeführten Weiterverwendungen des Materials die Einwilligung des jeweiligen Rechteinhabers einzuholen.

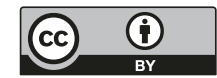

